\title{
Primary Nongestational Choriocarcinoma of the Ovary
}

\author{
A. M. M. Shariful Alam ${ }^{1}$, Syeda Nurjahan Bhuiyan ${ }^{2}$, Md. Rashid Un Nabi ${ }^{3}$ \\ Received: June 15, 2012 Accepted: October 10, 2013
}

\begin{abstract}
Pure primary ovarian choriocarcinoma is an extremely rare and aggressive tumor. It can be of gestational or nongestational in origin. The gestational type can arise from an ovarian pregnancy or can be of metastatic origin from uterine choriocarcinoma. The nongestational type is a very rare germ cell neoplasm. It is important to distinguish between two types of choriocarcinomas as nongestational origin is highly malignant and has worse prognosis than gestational type. But it is very difficult to differentiate by routine histological examination. Nongestational choriocarcinoma has been found to be resistant to single agent chemotherapy. It occurs usually around 13 years of age and is mainly confined to females under 20. Here we report a case of primary pure nongestational choriocarcinoma of the ovary in an unmarried girl of 14 years, diagnosed in 2001 and treated successfully with surgery and combination chemotherapy and remained disease-free till last reporting in September 2013.

Key words: Nongestational, Choriocarcinoma, Ovary, Chemotherapy
\end{abstract}

J Enam Med Col 2014; 4(1): 56-59

\section{Introduction}

Pure primary ovarian choriocarcinoma accounts for less than $1 \%$ of ovarian tumors. ${ }^{1}$ Shiromizu et al ${ }^{2}$ recently studied 467 ovarian germ cell tumors and found only one choriocarcinoma. Choriocarcinoma is an aggressive tumor that may occur during or without pregnancy. Gestational choriocarcinoma of the ovary can be primary, associated with ovarian pregnancy, or metastatic, arising from a primary gestational choriocarcinoma in the uterus. Nongestational choriocarcinoma of the ovary can be pure, but is more frequently associated with other germ cell tumors. ${ }^{3}$

Primary ovarian choriocarcinoma arising from germ cell is an extremely rare occurence. ${ }^{4}$ Primary extrauterine choriocarcinoma is very rare and it is found mostly in the genital tract in patients with coincident or antecedent pregnancy. Primary nongestational ovarian choriocacarcinomas (NGCOs) are usually seen with other germ cell tumors. ${ }^{5}$ Pure primary nongestational ovarian choriocarcinoma of the ovary is extremely rare. ${ }^{6}$ Choriocarcinoma of the ovary is a highly malignant ovarian tumor which is characterized pathologically by the presence of trophoblastic malignant cells, and biochemically by the production of the pregnancy hormone human chorionic gonadotropin (hCG) in absence of pregnancy. Choriocarcinoma is a rapidly invasive, widely metastatic, human chorionic gonadotropin (hCG) producing neoplasm which usually has an intrauterine location. ${ }^{7}$

Choriocarcinoma tends to be invasive and metastasize early and widely through both the venous and lymphatic systems. This disease is classified into two types in origin, gestational choriocarcinoma and nongestational germ cell tumor. Nongestational pure choriocarcinoma is so rare that the prognosis, chemo-sensitivity, and its genetic analysis have not been decided compared with that of gestational type. It is necessary, but difficult to distinguish nongestational choriocarcinoma from

1. Professor, Department of Clinical Oncology, Enam Medical College \& Hospital, Savar, Dhaka

2. Former Professor, Department of Obstetrics \& Gynecology, Chittagong Medical College \& Hospital, Chittagong

3. Consultant Radiation Oncologist, United Hospital Ltd., Dhaka

Correspondence A. M. M. Shariful Alam,Email: nicrh2002@gmail.com 
gestational choriocarcinoma except by DNA analysis. $^{8}$

The gestational type includes an ovarian metastasis from primary uterine choriocarcinoma which occurs in association with a normal pregnancy or spontaneous abortion, complete hydatidiform mole, or partial mole, and primary gestational ovarian choriocarcinoma which arises from ectopic pregnancy in the ovary. The nongestational type is a component of a mixed germ cell tumor and a pure ovarian choriocarcinoma is a very rare malignant tumor. ${ }^{8}$

\section{Case report}

A 14-year-old unmarried girl presented to Radiotherapy department, Chittagong Medical College \& Hospital on 16.06.2001 with the complaints of amenorrhea for last three months, lower abdominal pain with low-grade fever and vomiting of one-month duration. On examination, she was febrile and anemic. Abdominal examination revealed a diffuse soft non-tender mass of 24-week size. Her hemoglobin level was $9.0 \mathrm{gm} / \mathrm{dL}$. Ultrasound examination showed an abdomino-pelvic complex solid mass measuring $20 \times 20 \times 14 \mathrm{~cm}$ with mixed echogenicity. The uterus was normal in size, shape and echotexture. Intrauterine or ectopic pregnancy was ruled out. No focal lesion was seen in the uterus. There was no fluid in the pouch of Douglas (POD). The urine for pregnancy test was positive. Serum $\beta$-hCG level was $>5000 \mathrm{mIU} / \mathrm{mL}$. On exploratory laparotomy, there was a big necrotic, hemorrhagic, soft and friable mass of about $20 \times 20 \mathrm{~cm}$ in size occupying whole of the lower abdomen, arising from left ovary and adherent posteriorly with sigmoid colon. Uterus was bulky. Right fallopian tube and ovary were normal. Omentum was also adherent with the tumor. Tumor was separated from the omentum, intestine and colon. Total abdominal hysterectomy with bilateral salphingoophorectomy with omental biopsy was performed on 18.06.2001. In the immediate postoperative period serum $\beta$-hCG level was $>4000 \mathrm{mIU} / \mathrm{mL}$. Histological examination of the left ovarian mass revealed extensive hemorrhage, necrosis and clusters of malignant trophoblastic cells with enlarged, hyperchromatic and bizarre nuclei. No other germ cell element was detected. Omental biopsy showed no metastases. The patient was put on chemotherapy consisting of bleomycin $(15 \mathrm{mg}$ [D1-D3]), etoposide $\left(100 \mathrm{mg} / \mathrm{m}^{2}\right.$ [D1-D3]) and cisplatinum $\left(100 \mathrm{mg} / \mathrm{m}^{2}\right.$ [D1]) at 4 -week interval. She received 6 cycles of above chemotherapy up to October 2001 and was kept on follow-up after physical examination and relevant investigations. There were no signs of residual disease and serum $\beta$-hCG level was $<2.13 \mathrm{mIU} / \mathrm{mL}$.

\section{Discussion}

Women over the age of 40 are at increased risk for gestational choriocarcinoma. Nongestational choriocarcinoma arises in women under 40 years of age, and the frequency is reported less than $0.6 \%$ of all ovarian tumors. ${ }^{8}$ Goswami et $\mathrm{al}^{9}$ reported the mean age $13.6 \pm$ 6.9 years. Ovarian germ cell tumors (OGCTs) arise primarily in young women between 10 and 30 years of age. ${ }^{10}$ In a review of the literature on both mixed and pure primary choriocarcinoma of the ovary, the age range of the patients was seven months to 35 years with a mean of about 13 years. ${ }^{11}$ Most ovarian choriocarcinomas are gestational in origin, and usually are metastases from uterine or tubal choriocarcinomas. ${ }^{5}$ Primary ovarian choriocarcinomas are rarely aggressive tumors, and comprise only $1 \%$ of all ovarian tumors. ${ }^{12}$ They can present in the pure form, or may occur in association with other germ cell tumors like teratoma, dysgerminoma or yolk sac tumor. ${ }^{13}$ Pure ovarian choriocarcinoma can be of gestational or nongestational type. Gestational ovarian choriocarcinomas occur in women of reproductive age, and are often associated with normal or molar pregnancies. ${ }^{7}$ Our reported case is an unmarried 14-year-old girl who presented with pain in the lower abdomen and with an abdomino-pelvic mass, with history of amenorrhea. Though the patient presented with a history of amenorrhea of three months duration, relevant investigations failed to reveal any evidence of pregnancy or hydatidiform mole. The tumor did not reveal evidence of any other germ cell malignancy to determine the cell of origin. For an ovarian choriocarcinoma to be unequivocally derived from germ cells, it should be diagnosed in a nonpregnant woman or prepubertal girl. ${ }^{6}$ With no evidence of ovarian, tubal or uterine pregnancy, it is difficult to establish the present case as ovarian gestational choriocarcinoma or a metastasis from a uterine or tubal choriocarcinoma that has regressed. It is 
important to distinguish between gestational and nongestational choriocarcinomas, since nongestational choriocarcinomas have been found to be resistant to single agent chemotherapy, have a worse prognosis, and therefore require aggressive combination chemotherapy. ${ }^{9}$ The reasons for poorer prognosis have not yet been determined. The diagnostic criteria used to diagnose nongestational choriocarcinoma which were first described by Saito et $\mathrm{al}^{14}$ are mentioned below.

1. Absence of disease in the uterine cavity

2. Pathological confirmation of disease

3. Exclusion of molar pregnancy

4. Exclusion of coexistence of intrauterine pregnancy

According to these criteria, the present case could be diagnosed as nongestational choriocarcinoma, but Jacobs et $\mathrm{al}^{15}$ stated that a nongestational type of ovarian choriocarcinoma can be diagnosed with certainty only in a patient who is sexually immature, unable to conceive, or has never had sexual intercourse. With such stringent criteria, our case cannot be defined as nongestational choriocarcinoma. Distinction of nongestational choriocarcinoma from gestational choriocarcinoma is impossible on histomorphology unless an evidence of pregnancy or other germ cell neoplasms is encountered. There are no ultrastructural or immunohistochemical features unique to either. DNA analysis for identification of paternal sequences establishes the diagnosis of gestational or nongestational choriocarcinoma. $5,16,17$

DNA polymorphism analyses using two or three appropriate VNTR (a variable number tandem repeats is a location in a genome where a short nucleotide sequence is organized as tandem repeats) loci from the tumor cells reveals the detectable alleles of VNTR. This indicates that the genetic background of the tumor cells is identical to that of the patient, thereby establishing the diagnosis of nongestational choriocarcinoma. Only few cases of nongestational choriocarcinoma have so far been diagnosed by genetic analyses. An extensive literature search including Medline demonstrated only five reported cases of nongestational ovarian pure choriocarcinoma diagnosed with DNA polymorphic analysis from 1985 to $2007 .^{5,8}$ In the absence of such a facility, our case was diagnosed as nongestational type of primary ovarian choriocarcinoma. Choriocarcinoma is often diagnosed by the finding of an elevated hCG level in association with metastatic lesion detected radiographically. The level of serum or urine beta-hCG is a good tumor marker for the progression or remission of disease. ${ }^{8}$

Modern treatment of nongestational choriocarcinoma of the ovary depends on surgical excision and a choice of combination chemotherapy based upon thorough histological evaluation of all the elements present. ${ }^{18,19}$ Gestational choriocarcinoma is treated with methotrexate-based chemotherapy, for example MEA (methotrexate, etoposide and actinomycin-D), EMA/CO (methotrexate, etoposide, actino-mycin-D, cyclophosphamide and vincristine), or EP/EMA (etoposide, cisplatin, methotrexate and actinomycin-D). However, nongestational ovarian choriocarcinoma (germ cell tumor) is so rare that there is lack of information on therapeutic options. Germ cell tumors of the ovary are treated with total abdominal hysterectomy and bilateral salpingooophorectomy. A complete staging operation is indispensable for management and prognostication. In young patients, stage I germ cell tumors can be treated with conservative surgery, i.e., unilateral oophorectomy or salpingo-oophorectomy. Postoperative chemotherapy is recommended by combination chemotherapy with the BEP (bleomycin, etoposide and cisplatin) or methotrexate-based regimen. ${ }^{8}$ The present case was treated with bleomycin, etoposide and cisplatin based combination chemotherapy after total abdominal hysterectomy and bilateral salphingoophorectomy and obtained a very good response. The prognosis of gestational choriocarcinoma is getting better by advances of combination chemotherapy. The survival rate has increased to $96.4 \%$ in 15 years since $1985 .^{8}$

Nongestational pure choriocarcinoma of the ovary is so rare that it is not known whether the prognosis is worse than gestational choriocarcinoma. Our reported case seems to have a good prognosis as she was in good health till the last follow-up (September 2013), and leading a normal life without any evidence of residual or recurrent disease. Some papers reported that nongestational choriocarcinoma of the ovary has worse prognosis and is less sensitive to methotrexate-based chemotherapeutic regimens compared to gestational neoplasm. But they did 
not diagnose definitely by DNA polymorphism analysis. It is important to clarify whether the tumor arose from a gestational or nongestational origin in order to understand the prognosis of this disease accurately. ${ }^{8}$

Although primary ovarian nongestational choriocarcinoma is exceedingly rare and definitive treatment of it is not yet well defined, in some cases it may have a very good outcome following surgery with adjuvant BEP schedule with proper hematological and supportive care.

\section{References}

1. Scully RE. Tumors of the ovary and maldeveloped gonads. In: Hartmann WH (ed). Atlas of tumor pathology. Washington, DC. Armed Forces Institute of Pathology 1979: 243-245.

2. Shiromizu K, Kawana T, Sugase M, Izumi R, Mizuno M. Clinicostatistical study of ovarian tumors of germ cell origin. Asia Oceania J Obstet Gynaecol 1991; 17: 207-215.

3. Talerman A. Germ cell tumors. Curr Top Pathol 1992; 85: 165-202.

4. Park SH, Park A, Kim JY, Kwon JH, Koh SB. A case of non-gestational choriocarcinoma arising in the ovary of a postmenopausal woman. J Gynecol Oncol 2009; 20(3): 192-194.

5. Tsujioka H, Hamada H, Miyakawa T, Hachisuga T, Kawarabayashi T. A pure nongestational choriocarcinoma of the ovary diagnosed with DNA polymorphism analysis. Gynec Oncol 2003; 89(3): 540-542.

6. Raju GC, Woo J, Marchack D, Narayansingh V. Primary nongestational choriocarcinoma of the ovary. Postgraduate Medical Journal 1985; 61: 757-758.

7. Liu Z, Mira JL, Cruz-Caudillo JC. Primary gastric choriocarcinoma. Arch Pathol Lab Med 2001; 125(12): 1601-1604.

8. Yamamoto E. Ovary: choriocarcinoma. Atlas Genet Cytogenet Oncol Haematol 2008. Available at:
http://AtlasGeneticsOncology.org/tumors/ovarychor iocarcID5219.html. Accessed March 2012.

9. Goswami D, Sharma K, Zutshi V, Tempe A, Nigam S. Nongestational pure ovarian choriocarcinoma with contralateral teratoma. Gynecol Oncol 2001; 80(2): 262-266.

10. Zalel Y, Piura B, Elchalal U, Czernobilsky B, Antebi S, Dgani $\mathrm{R}$ et al. Diagnosis and management of malignant germ cell ovarian tumors in young females. Int $\mathrm{J}$ Gynaecol Obstet 1996; 55: 1-10.

11. Fox H, Langley FA. Tumours of the ovary. London: Heinemann, 1976

12. Gangadharan VP, Mathew BS, Kumar KS, Chitrathara K. Primary choriocarcinoma of the ovary. Indian J Cancer. 1999; 36: 213-215.

13. Pai MR, Naik R. Nongestational choriocarcinoma of the ovary - a case report. Indian J Pathol Microbiol 1995; 38(3): 317-318.

14. Saito M, Azuma T, Nakamura K. On ectopic choriocarcinoma. World Obstet Gynecol 1963; 17: 459-484.

15. Jacobs AJ, Newland JR, Green RK. Pure choriocarcinoma of the ovary. Obstet Gynecol Surv 1982; 37: 603-609.

16. Yamamoto E, Ino K, Yamamoto $T$, Sumigama S, Nawa A, Nomura $\mathrm{S}$ et al. A pure nongestational choriocarcinoma of the ovary diagnosed with short tandem repeat analysis. Case report and review of literature. Int $\mathrm{J}$ Gynecol Cancer 2007; 17(1): 254-258.

17. Koo HL, Choi J, Kim KR, Kim JH. Pure non-gestational choriocarcinoma of the ovary diagnosed by DNA polymorphism analysis. Pathol Int 2006; 56(10): 613-616.

18. Wider JA, Marshall JR, Bardin CW, Lipsett MB, Ross GT. Sustained remission after chemotherapy for primary ovarian cancers containing choriocarcinoma. N Engl J Med 1969; 280: 1439.

19. Gerbie MV, Beever JI, Jamini H. Primary choriocarcinoma of the ovary. Obstetrics \& Gynecology 1975; 46: 720 . 